\title{
Is there a flatness problem in classical cosmology?
}

\author{
Phillip Helbig ${ }^{\star}$ \\ Thomas-Mann-Str. 9, D-63477 Maintal, Germany
}

Accepted 2011 December 05. Received 2011 December 04; in original form 2011 November 27

\begin{abstract}
I briefly review the flatness problem within the context of classical cosmology and examine some of the debate in the literature with regard to its definition and even the question whether it exists. I then present some new calculations for cosmological models which will collapse in the future; together with previous work by others for models which will expand forever, this allows one to examine the flatness problem quantitatively for all cosmological models. This leads to the conclusion that the flatness problem does not exist, not only for the cosmological models corresponding to the currently popular values of $\lambda_{0}$ and $\Omega_{0}$ but indeed for all Friedmann-Lemaître models.
\end{abstract}

Key words: cosmology: theory - cosmological parameters.

\section{INTRODUCTION}

The flatness problem has been called one of the outstanding puzzles in cosmology (e.g. Dicke \& Peebles 1979). This in itself is rather puzzling in view of the fact that the arguments in favour of it being a problem are rather vague and heuristic, while quantitative arguments have been presented against the claim that it is a problem, at , least for some classes of cosmological models (e.g. Coles \& Ellis 1997; Lake 2005). The flatness problem is one of the main motiva'tions for the inflationary scenario (Guth 1981). Of course, if there is no flatness problem (or, indeed, even if there were no motivation at all for inflation), this does not mean that inflation could not ' have occurred. However, it does mean that inflation should not be taken as given based on the belief that it explains away the flatness problem and thus without it classical cosmology leads to absurd conclusions.

The plan of this paper is as follows. In Section2I present the basic equations needed in the rest of the paper, mainly to define my notation (unfortunately, there is not a uniform notation in the literature) and give an overview of the entire cosmological parameter space relevant to the discussion. Section 3 gives a brief historical overview of the flatness problem and some qualitative arguments against it. In Section 4 I discuss a new quantitative argument regarding cosmological models which will collapse in the future. Sections 5 and 6 discuss previous quantitative results by others for other classes of cosmological models. Section 7 summarizes the results for all cosmological models.

\section{BASIC COSMOLOGY}

I assume that, at the level of detail necessary, the universe can be described by the Friedmann-Lemaitre equation

* E-mail: helbig @astro.multivax.de

$$
\dot{R}^{2}=\frac{8 \pi G \rho R^{2}}{3}+\frac{\Lambda R^{2}}{3}-k c^{2}
$$

with the dimensionless constant $k$ equal to $-1,0,+1$ depending on spatial curvature (negative, vanishing or positive, respectively); $R$ is the scale factor (with dimension length) of the universe, $G$ the gravitational constant, $\rho$ the density, $\Lambda$ the cosmological constant (dimension time $^{-2}$ ) and $c$ the speed of light. It is useful to define the following quantities:

$$
\begin{aligned}
H & :=\frac{\dot{R}}{R} \\
\lambda & :=\frac{\Lambda}{3 H^{2}} \\
\Omega & :=\frac{\rho}{\rho_{\text {crit }}} \equiv \frac{8 \pi G \rho}{3 H^{2}} \\
K & :=\Omega+\lambda-1 \\
q & :=\frac{-\ddot{R} R}{\dot{R}^{2}} \quad \equiv \frac{-\ddot{R}}{R H^{2}} \equiv \frac{\Omega}{2}-\lambda
\end{aligned}
$$

which are all dimensionless except that $H$ has the dimension time $^{-1} . H$ is the Hubble constant, $\lambda$ the normalized cosmological constant, $\Omega$ the density parameter, $k=\operatorname{sign}(K)$ and $q$ is the deceleration parameter 11 For $\lambda=0$ and $k=0, \rho=\rho_{\text {crit }}=\frac{3 H^{2}}{8 \pi G}$. This density is 'critical' in the sense that, for $\lambda=0$, a greater (lesser) density implies a positive (negative) curvature and a universe (assumed to be expanding now) which will collapse in the future (expand forever); similarly, for $k=0$, a greater (lesser) density implies a negative (positive) cosmological constant and a universe (assumed to be expanding now) which will collapse in the future (expand forever). However, in the general case $(k \neq 0$ and $\lambda \neq 0)$,

\footnotetext{
${ }^{1} q>0$ implies that the universe is decelerating; the minus sign is included in the definition since at the time of its invention it was assumed that $\lambda=0$ which implies that $q>0$ (or $q=0$ in the case of a universe devoid of matter).
} 
$\rho_{\text {crit }}$ doesn't have any special meaning, though $\Omega$ remains a useful parameter. Equation 11 can be rearranged, using the definitions above, to give

$R=\frac{c}{H} \frac{\operatorname{sign}(K)}{\sqrt{|K|}}$

thus $R$ is positive for $k=+1$ and negative for $k=-1$; for $k=0$, $R$ can be defined as $\frac{c}{H}$.

It can be useful to express equation (1) with the values of the dimensionless parameters as observed now, denoted by the suffix 0 . This leads to

$\dot{R}^{2}=\dot{R}_{0}^{2}\left(\frac{\Omega_{0} R_{0}}{R}+\frac{\lambda_{0} R^{2}}{R_{0}^{2}}-K_{0}\right)$

or, making use of the definition of $H$,

$H^{2}=H_{0}^{2}\left(\frac{\Omega_{0} R_{0}^{3}}{R^{3}}+\lambda_{0}-\frac{K_{0} R_{0}^{2}}{R^{2}}\right)$.

(Note that, with

$z:=\frac{R_{0}}{R}-1$

this leads to

$\frac{\mathrm{d} z}{\mathrm{~d} t}=\frac{\mathrm{d} z}{\mathrm{~d} R} \frac{\mathrm{d} R}{\mathrm{~d} t}=\frac{\mathrm{d} z}{\mathrm{~d} R} \dot{R}=-H_{0}(1+z) \sqrt{F(z)}$

where

$F(z)=\Omega_{0}(1+z)^{3}-K_{0}(1+z)^{2}+\lambda_{0}$

which is the starting point for calculating light-travel time and distance as a function of redshift.) In general, $H, \lambda$ and $\Omega$ all change with time. Note that since

$\lambda=\lambda_{0}\left(\frac{H_{0}}{H}\right)^{2}$,

the change in $\lambda$ with time is due entirely to the change in $H$ with time, since $\Lambda$ is constant. Also, since the density $\rho$ is inversely proportional to the cube of $R$,

$\Omega=\Omega_{0}\left(\frac{H_{0}}{H}\right)^{2}\left(\frac{R_{0}}{R}\right)^{3}$,

the variation in $\Omega$ is due both to variation in $H$ and to the decrease in density as the universe expands.

In general, $\lambda$ and $\Omega$ evolve with time. (They do not for the $(\lambda, \Omega)$ values of $(0,0)$ (Milne model), $(0,1)$ (Einstein-de Sitter model), $(1,0)$ (de Sitter model) and for the static Einstein model [in which $\lambda$ and $\Omega$ are infinite (though $\Lambda$ and $\rho$ are not; $\Lambda=4 \pi G \rho$ ) since $H$ is 0$]$.) For an excellent discussion of the evolution of $\lambda$ and $\Omega$ (though expressed in the older notation using $\sigma=\frac{\Omega}{2}$ and $q=\sigma-\lambda$ ), see Stabell \& Refsdal (1966). For a classification based on the evolution of $\lambda$ and $\Omega$ using the more modern notation used in the present paper, see table 1, fig. 1 and section a.i. in Helbig (1996) 2 For the present discussion, the basic information needed can be seen in Fig. 11[for derivation, see Stabell \& Refsdal (1966)] which shows an overview of the $\lambda-\Omega$ plane. The vertical line corresponds to $\lambda=0$; the diagonal line corresponds to $k=0$ with $k=-1$ below it and $k=+1$ above it. The curve near the vertical line [corresponding to the A1 curve in Stabell \& Refsdal (1966)]

2 This is the arXiv version of Kavser, Helbig \& Schramm
$\begin{aligned} & \text { 1997) but in contrast to the A\&A paper con- } \\ & \text { tains the User's Guide as well; also available at }\end{aligned}$

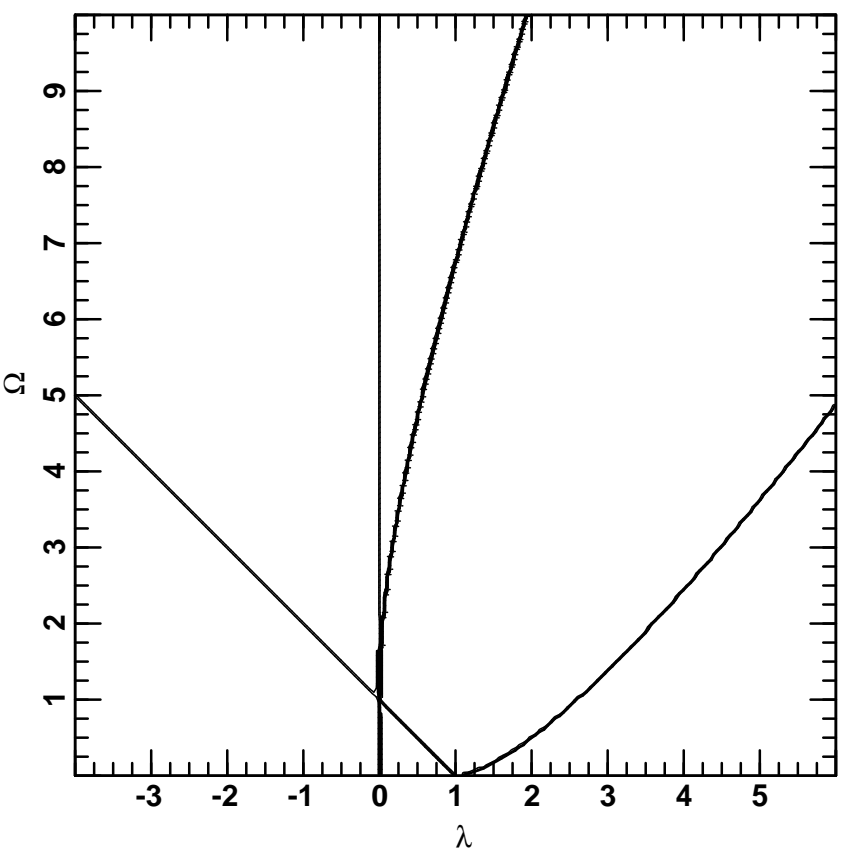

Figure 1. The $\lambda-\Omega$ plane. See text for details.

separates models which will collapse (to the left) from those which will expand forever (to the right). Models on the curve start arbitrarily close to the Einstein-de Sitter model (like all non-empty big-bang models) and asymptotically approach the static Einstein model which has $\lambda=\Omega=\infty$ (since $H=0 ; \Lambda$ and $\rho$ have finite values). The other curve [corresponding to the A2 curve in Stabell \& Refsdal (1966)] separates big-bang models (to the left) from non-big-bang models (to the right); the latter contract from an infinite to a finite size then expand forever. Models on the curve start at the static Einstein model and asymptotically approach the de Sitter model (the latter feature is shared with all models which expand forever and have $\lambda>0$ ). Fig. 2 shows some sample trajectories in the $\lambda-\Omega$ parameter space superposed in Fig. 1 Note that all the lines and curves in Fig. 1 correspond to trajectories. In addition, there are models at the intersections of the lines with $(\lambda, \Omega)$ values of $(0,0)$ (the Milne model), $(0,1)$ (the Einstein-de Sitter model), $(1,0)$ (the de Sitter model) and at $(\infty, \infty)$ (the Einstein model); in these, $\lambda$ and $\Omega$ are constant in time. Also, note that the trajectories do not cross; this means that the history of a cosmological model (i.e. the way $\lambda$ and $\Omega$ change with time) is completely determined by the values at any point on it (in practice, by measuring the values at the present time, $\lambda_{0}$ and $\Omega_{0}$ ). Since the lines and curves are also valid trajectories, this means that the signs of $\lambda$ and $K$ cannot change and that a model with $\Omega=0$ at any time has $\Omega=0$ at all times.

Fig. 3 shows contours of constant $H t$, i.e. the age of the universe in units of the Hubble time $\left(H^{-1}\right)$. The relation between these contours and the trajectories shown in Fig. 2 is important for the discussion in Sections 4 and 6 Note that this is a smooth and well behaved function of $\lambda$ and $\Omega$, independent of the geometry (global curvature), origin and fate (big bang or not, recollapse or eternal expansion) or contents (matter, cosmological constant) of the universe (except of course that the age of the universe is infinite on the A2 curve; contours to the right of the A2 curve indicate the time
/singe the minimaums of the scale factor). 


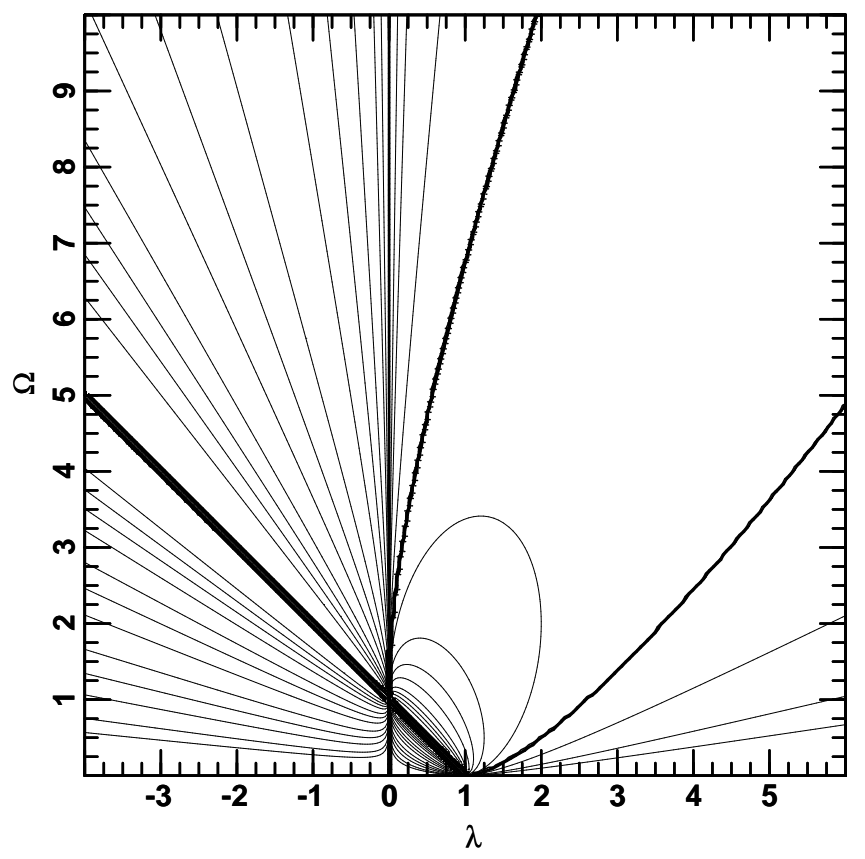

Figure 2. Evolutionary trajectories in the $\lambda-\Omega$ plane.

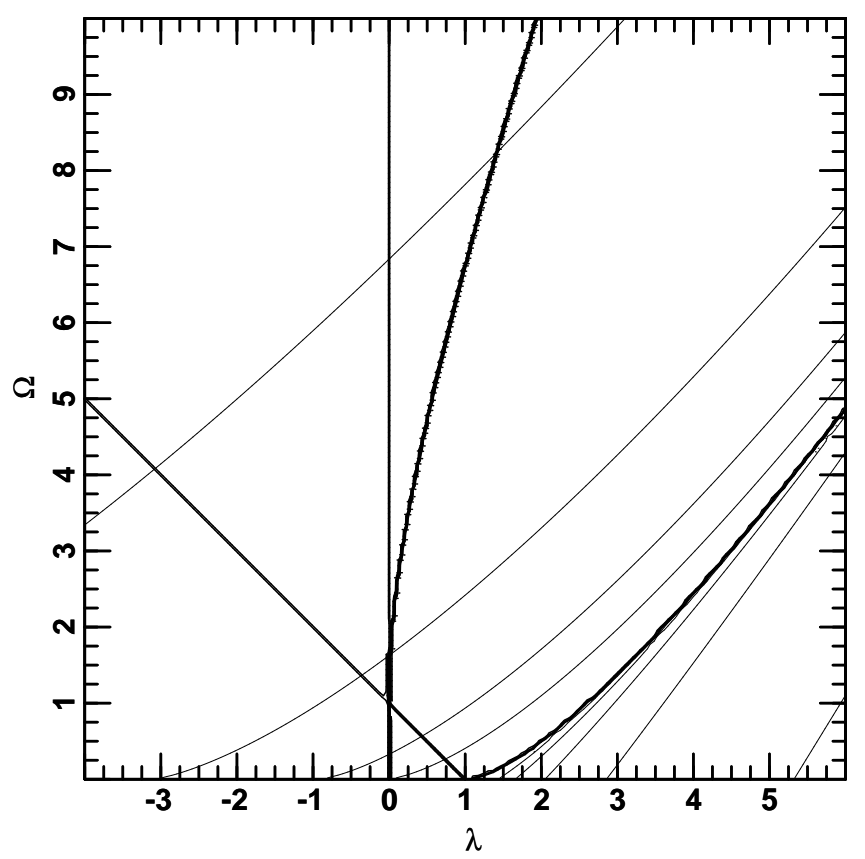

Figure 3. The age of the universe in units of $H^{-1}$. From upper left to lower right, contours are at $0.4,0.6,0.8,1.0,1.0,0.8,0.6,0.4$ and 0.2 Between the two contours at 1.0 is the A2 curve which corresponds to $\infty$. To the left of the curve the contours indicate the time since the big bang; to the right they indicate the time since the universe started expanding from its minimum size.

\section{A BRIEF HISTORY OF THE FLATNESS PROBLEM}

The flatness problem appears in two forms. One states that if $\Omega \approx 1$ today, then in the early universe it was arbitrarily close to 1 ; the assumption is that some 'mechanism' is needed to explain this 'finetuning' (e.g. Guth 1981). (It is usually not stated but almost always assumed that no fine-tuning would be necessary if $\Omega$ were not $\approx 1$ today.) The other states that if $\Omega$ changes with time, then we should be surprised that $\Omega$ is (still) $\approx 1$ today (e.g. Lake 2005). In other words, the problem is that the mechanism whereby $\Omega$ is forced to be $\approx 1$ is unknown. Solving one of these variants of the flatness problem does not necessarily solve the other variant.

[Historically, the flatness problem was first discussed during a time when $\lambda$ was thought to be zero. Thus, most discussions took this as given. As mentioned above, in this case $\Omega=1$ corresponds to $k=0$, i.e. a flat universe, hence the name 'flatness problem' for the question why the universe is (nearly) flat today considering that $\Omega$ evolves away from $\Omega=1(k=0)$ with time. If $\lambda$ is not constrained to be zero, then the flatness problem should be re-phrased as the Einstein-de Sitter problem, i.e. the question is why the universe is (in some sense) close to the Einstein-de Sitter model (which is an unstable fixed point and a repulsor) today when $|\lambda|$ and $\Omega$ can take on values between 0 and $\infty$. However, for brevity I will continue to use the term 'flatness problem' even for the more general case and sometimes mention only the change in $\Omega$ with time. Nevertheless, it is important to keep in mind (though it doesn't change the thrust of the argument in all cases) that the discussion should really be about the Einstein-de Sitter problem.]

One possible 'solution' to the flatness problem is simply to claim that $\Omega \equiv 1$. In this case (for $\lambda=0$ ), since $\Omega$ doesn't evolve with time, no explanation is needed as to why $\Omega \approx 1$ today; in other words, we don't have to worry about living at a special time. Of course, the fact that $\Omega$ just happens to be 1 is thought by some to be improbable in some ill-defined sense, so it is more convincing if there is some mechanism, such as inflation, which produces $\Omega=1$ to at least a very good approximation, rather than having to rely on the lucky coincidence of living in a cosmological model in which $\Omega$ does not change with time. (Of course, the Einstein-de Sitter model is now ruled out by observations. Before this was the case, the argument ' $\Omega$ must be exactly 1 because if not it will evolve away to to an arbitrarily large or arbitrarily small value' was indeed used.) However, this solution does not work in practice, as has been pointed out by Guth (1981) and Coles \& Ellis (1997): even if our universe is described by the Einstein-de Sitter model when averaged over large scales, this is obviously not the case on smaller scales; any deviations from the Einstein-de Sitter model would then grow with time, even if the entire universe were still, on average, described by the Einstein-de Sitter model, so that we would not expect our observable universe to be described exactly by the Einstein-de Sitter model at a 'random' time.

Note that a flat universe $(k=0, \Omega+\lambda=1)$ doesn't really offer much of an advantage. Though it is true that if $\Omega+\lambda=1$ then this equation always holds, while in general the sum is timedependent, the same argument can be applied to the value of $\Omega$ in the flat case as in the case of $\lambda=0$. In other words, although it is true that in a flat universe $\Omega+\lambda$ doesn't change with time, $\Omega$ itself suffers from the same 'problem' it does in the $\lambda=0$ case (except in the special cases of the Einstein-de Sitter model and the de Sitter model). Thus, for the flatness problem in the broader sense of the term (i.e. understanding why $\Omega \approx 1$ today), the cosmological model favoured by observations $\left(\lambda_{0} \approx 0.7, \Omega_{0} \approx 0.3, k=0\right.$ to very good precision) is actually worse than the Einstein-de Sitter model, even if it were exactly flat.

Another solution discussed by Coles \& Ellis (1997) is to deny that it is a problem that we live at some special time in the history of the universe, since it is not equally likely that we could live at any time; this is of course the weak anthropic principle. While the weak anthropic principle certainly plays some role in determining the probability of observing given values of the cosmological parameters, it is not obvious that it can quantitatively explain why our 
universe is so close to the Einstein-de Sitter model. In any case, most people would probably prefer an explanation which doesn't rely on the anthropic principle. I shall return to the weak anthropic principle in Section 6. [Note that a perfectly spatially flat universe is always perfectly spatially flat, and thus does not suffer from the 'special time' problem with regard to flatness (though one might need some explanation for the perfect flatness itself). However, it still suffers from the Einstein-de Sitter problem (except in the cases of the Einstein-de Sitter universe and the de Sitter universe in which there is no evolution of $\lambda$ and $\Omega$ ).]

One definition of a 'special time' is a time such that the radius of curvature is comparable to the distance to the (particle or event) horizon or the radius of the Hubble sphere. Since in general these quantities are not simply proportional and evolve differently with time, it could be seen as a coincidence (possibly needing explanation) if they are comparable. A perfectly flat universe with an infinite radius of curvature doesn't have this problem since the radius of curvature is always infinite. However, this 'advantage' of a flat universe is not so much a solution to the time-scale problem as a statement that it doesn't exist (with respect to the evolution of the curvature radius) in such a universe; one could still ask what 'causes' the universe to be spatially flat. Also, as noted above, a spatially flat universe still suffers from the 'Einstein-de Sitter problem'. (This coincidence problem is similar to, but distinct from, the flatness problem. If at all, it exists only for $\lambda=0$ and $\Omega<1$. I plan to discuss this in a future paper. As is shown below, this class of models is somewhat atypical with respect to the flatness problem; this is also the case for the coincidence problem. Since $\lambda=0$ was a common assumption when these problems were first discussed, sometimes particular features of this class of models are mistaken for generic features of Friedmann-Lemaitre models.)

These three 'solutions' to the flatness problem $-\Omega \equiv 1$ (and $\lambda \equiv 0), k=0$, anthropically selected special time - are thus unsatisfactory. Are there any satisfactory ones?

\subsection{The qualitative flatness problem: Is a fine-tuning of initial conditions required?}

The flatness problem is often presented as a fine-tuning problem (e.g. Guth 1981): if $\Omega$ is near 1 to day, then at some time $t_{\text {fine }}$ in the past it must have been 1 to a very high accuracy. I refer to this sense of the flatness problem as the 'qualitative flatness problem'. This argument is completely bogus, as has been pointed out by many authors (e.g. Coles \& Ellis 1997; Lake 2005): all non-empty models begin their evolution at the Einstein-de Sitter model, so of course the further back in time one goes, the 'more finely tuned' $\Omega$ is. The point is, within the context of classical cosmology, there is nothing special about a time $t_{\text {fine }}$ chosen so that $\Omega$ is very close to 1 at that point. Times such as the Planck time are often used in examples of the flatness problem, but not only is the Planck time irrelevant within purely classical cosmology but also there is no known theory which predicts the likelihood distribution of $\Omega$ values at this (or any other) time. This should be obvious from the fact that for any value of $\Omega$ today, one can choose a time $t_{\text {fine }}$ such that $\Omega$ is as close to 1 as desired. In other words, the flatness 'problem' would still exist if $\Omega$ were appreciably greater or less than 1 today, only the time $t_{\text {fine }}$ for a given degree of fine tuning would occur at an earlier time. (Why this is less mysterious for many people is not clear to me.) Thus, the 'problem' still remains. (Also, the degree of fine tuning required for there to be a 'problem' is not well defined, but is arbitrary and subjective.) Alternatively, if the problem is seen as a problem connected with the observed value of $\Omega$, then the fact that it exists for many other values as well, indeed for all values, could lead to the conclusion that it is not a problem at all. This is thus a qualitative argument against the existence of the qualitative flatness problem.

Evrard \& Coles (1995) (see also Coles \& Ellis 1997) also point out that the assumption implicit in the qualitative flatness problem, namely that some wide range of $\Omega$ values are a priori equally likely at some early time, constitutes a prior which is incompatible with the assumption of minimal information. This can be regarded as a quantitative solution to the qualitative flatness problem (or, perhaps, an argument against its existence).

The qualitative flatness problem thus does not exist; it is merely a consequence of the way in which a universe, described by the Friedmann-Lemaître equation, evolves and how dimensionless observable quantities such as $\Omega$ are defined. Suppose one is standing at the bottom of the famous Leaning Tower of Pisa and observes a cannonball dropping past one's face to the ground. In some absolute sense it is travelling slowly (its speed is much less than the speed of light, say). One can measure the acceleration and calculate that, at a time $t_{\text {fine }}$ in the past, its velocity must have been extremely 'finely tuned' to almost zero. In fact, at a finite time in the past, its velocity was zero, and at that time it was at a particular place (say, the top of the tower). What's more, other objects which, due to the effects of air resistance, are falling past one at other speeds are all found to be 'finely tuned' so that their velocity was 0 at a given time in the past (and for all objects, the height at that time is the same).

Obviously, there is nothing at all puzzling about this scenario. Nevertheless, this is the type of 'evidence' which is often presented for the 'existence of the flatness problem', with $\Omega$ taking the place of velocity (being 1 at the beginning and not 0 , of course). Argument from analogy can be misleading, so one should put more faith in quantitative arguments.

In the above case, the solution is obviously to be found in the initial conditions: Galileo is dropping objects from the top storey. In fact, in the cosmological case the lack of a need for fine-tuning is even more obvious. While Galileo could give the objects an initial velocity rather than just dropping them, any non-empty bigbang universe described by the Friedmann-Lemaitre equation always begins arbitrarily close to the Einstein-de Sitter model. There is no need for fine-tuning since there is no possible range of values at the initial time. See Section 3.2 for the discussion of a somewhat better, but still flawed, analogy. Nevertheless, even if it is not a puzzle why $\Omega=1$ at early times, one can still ask whether we should be surprised that $\Omega \approx 1$ today.

\subsection{The quantitative flatness problem: Should we be surprised that $\Omega_{0} \approx 1$ ?}

What is the relation between the two forms of the flatness problem discussed above? Within the context of classical cosmology, the first statement, that the universe was arbitrarily close to the Einstein-de Sitter model near the beginning, is almost always true. (It is always true for non-empty big-bang models. For empty models, similar arguments apply: For $\lambda<1$, the starting point is not the Einstein-de Sitter model but rather the Milne model $(\lambda=\Omega=0)$. For $\lambda>1$ the starting point is the de Sitter model $(\lambda=1$ and $\Omega=0$ ), although these are not big-bang but rather bounce models. (The limiting case of the de Sitter model itself can be thought of as a big-bang model in which the big bang occurred in the infinite past.) Another non-big-bang model is the static Einstein model. At first sight, this model seems completely different than the Einstein- 
de Sitter model. However, mathematically both are unstable fixed points. Interestingly, Eddington (1930) argued that this is a mark against the Einstein model, since it is unstable (thus unlikely to hold in a realistic universe): the attraction due to gravity and the repulsion due to the cosmological constant are exactly balanced. Since the density of matter decreases if the universe expands and vice versa, it is unstable not only to changes in the value of the cosmological constant or the density per se, but also to departures from the static state. The Einstein-de Sitter universe is unstable in exactly the same sense: as long as it is not perturbed, the values of the cosmological parameters remain constant; if they are even slightly different, they evolve away from the pure Einstein-de Sitter state. Of course, in some sense this is not a problem since if we assume that the universe is exactly described by the FriedmannLemaitre equation, then the behaviour at all times is fixed; there is no way to perturb it. On the other hand, if only because the universe is not completely homogeneous, the Friedmann-Lemaître equation cannot be exact, so the objection is a valid one. Interestingly, the same argument is rarely used against the Einstein-de Sitter universe, even though mathematically both are unstable fixed points and hence suffer from the same weakness. [In fact, the argument is turned around: because the Einstein-de Sitter universe is unstable, if the observational constraints on the cosmological parameters describing our universe are compatible with the Einstein-de Sitter model, it is much more probable that our universe is described (almost) exactly by the Einstein-de Sitter model, rather than one nearby in the parameter space. Of course, the caveats regarding 'probable', 'almost' and 'nearby' mentioned above make this an invalid line of argument.] In both cases, a universe described exactly by the model in question would be stable (but would contain no cosmologists, since these presumably require inhomogeneities, and of course could not be perturbed from 'outside the universe') while a realistic universe would be at least locally unstable even if the model were an accurate description of a large-scale average. Of course, the static Einstein universe was ruled out observationally before the Einstein-de Sitter universe was, but while observational arguments themselves are essential, they should not influence what should be purely theoretical or mathematical arguments.) As discussed above, this can be interpreted to mean that this aspect of the flatness problem exists for all cosmological models (i.e. regardless of what values we observe for $\lambda_{0}$ and $\Omega_{0}$ ), which means that in this respect there is nothing special about our universe, or that it is not a problem at all, but just a consequence of definitions. Obviously, the second form of the problem (If $\Omega$ evolves, even to arbitrarily large values in a finite time, should we be surprised if it is $\approx 1$ ?) holds only if $\Omega \approx 1$ today; on the other hand, holding that the first form is not a problem (which, as outlined above, I am not alone in claiming) does not automatically solve the second problem. The rest of this paper is concerned mainly with the second form: should we be surprised that $\Omega \approx 1$ today? This 'quantitative flatness problem' is more subtle, but also has solutions within the context of classical cosmology.

An analogy often used in discussing the flatness problem is that of a tightrope walker (e.g. Coles \& Ellis 1997; Coles 2009): The Einstein-de Sitter model corresponds to the balanced tightrope walker. As long as he stays balanced, he will stay where he is. However, a slight deviation will grow, quickly bringing the tightrope walker far from the tightrope. If one walks by a circus tent at an arbitrary time and looks inside, one would expect to find the tightrope walker either balanced on the tightrope or on the ground after having fallen; it is extremely improbable that one would just happen to see him during his fall. The ground corresponds to one of the extreme values of $\Omega$ ( $\infty$ or 0 ). Every statement of the quantitative flatness problem essentially follows this example. Were it a valid analogy, then there would indeed be a quantitative flatness problem. In the following sections, I will show quantitatively why the analogy is wrong and hence why there is no quantitative flatness problem.

\section{COSMOLOGICAL MODELS WHICH COLLAPSE IN THE FUTURE: A NEW SOLUTION TO THE FLATNESS PROBLEM}

All cosmological models (assumed to be expanding now) with $\lambda<$ 0 will collapse in the future: $\ddot{R}$ is negative for all values of $R$ and for large $R$ is proportional to $R$. Models with $\lambda=0$ will collapse for $\Omega>1$. In addition, models with $\lambda>0$ will collapse provided that $\Omega>1$ (which in this case implies $K>0$, i.e. $k=+1$ ), $q>0$ and $\alpha<1$, where

$\alpha=\operatorname{sign}(K) \frac{27 \Omega^{2} \lambda}{4 K^{3}}$

(Stabell \& Refsdal 1966; Lake 2005). (The A1 and A2 curves mentioned above have $\alpha=1$.) In Fig. 1, these are in the area between $\lambda=0$ and the A1 curve. Empty big-bang models start arbitrarily close to the Milne model with $(\lambda, \Omega)$ values of $(0,0)$; non-empty big-bang models start arbitrarily close to the Einstein-de Sitter model with $(\lambda, \Omega)$ values of $(0,1)$. The evolution of $\lambda$ and $\Omega$ can be viewed as trajectories in the parameter space: $\lambda$ and $\Omega$ evolve from the starting point to infinity and return along the same trajectory. [For the definitive discussion, see Stabell \& Refsdal (1966); a very useful visualisation can be found at Leahy (2003).] Note that such trajectories do not intersect; this means that a trajectory is uniquely determined by measuring $\lambda$ and $\Omega$ at any one time (practical is of course doing so now).

The fact that $\lambda$ and $\Omega$ evolve to $\infty$ (and back) in a finite time immediately illustrates what is wrong with the tightrope-walker analogy for these models: the proper analogy for a universe which will collapse in the future would be a tightrope walker who, if he falls off the tightrope, doesn't stop when he hits the ground, but rather continues through the Earth and to infinity and back, finally approaching the tightrope from below until he is ultimately almost balanced again. It should be immediately obvious that in this case all values of $\lambda$ and $\Omega$ cannot be equally probable. Making the analogy more quantitative, it turns out that the tightrope walker actually spends most of his time between the rope and the floor, thus we should not be surprised to find him somewhere between the tightrope and the ground when we look inside the tent. In other words, there is no quantitative flatness problem in these models.

To quantify this, I have calculated the quotient of the age of the universe now and at the time of maximum expansion as a function of $\lambda$ and $\Omega$. The age of the universe is given by

$t=\int_{0}^{R(t)} \frac{\mathrm{d} R}{\sqrt{\dot{R}_{0}^{2}\left(\frac{\Omega_{0} R_{0}}{R}+\frac{\lambda_{0} R^{2}}{R_{0}^{2}}-K_{0}\right)}}$

which follows from equation (3). For the current age, the upper limit is given by equation (2); at the time of maximum expansion it is found by calculating the (smallest) zero of $\dot{R}^{2}$ (since $\dot{R}^{2}$ cannot be negative). This is shown in Fig. (4). It is clear that large values of $\lambda$ and $\Omega$ occur only during a relatively short time in the history of the universe, near the time of maximum expansion (at the precise 


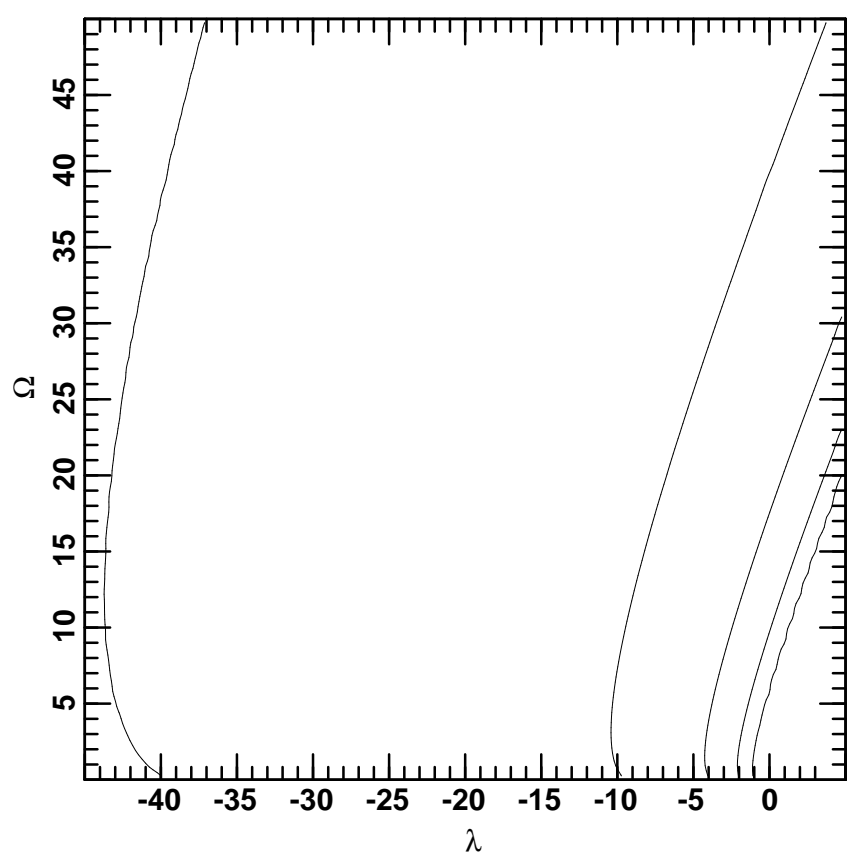

Figure 4. The age of the universe as a fraction of the time between the big bang and maximum expansion. Contours, from right to left, are at $0.5,0.6$, $0.7,0.8$ and 0.9 .

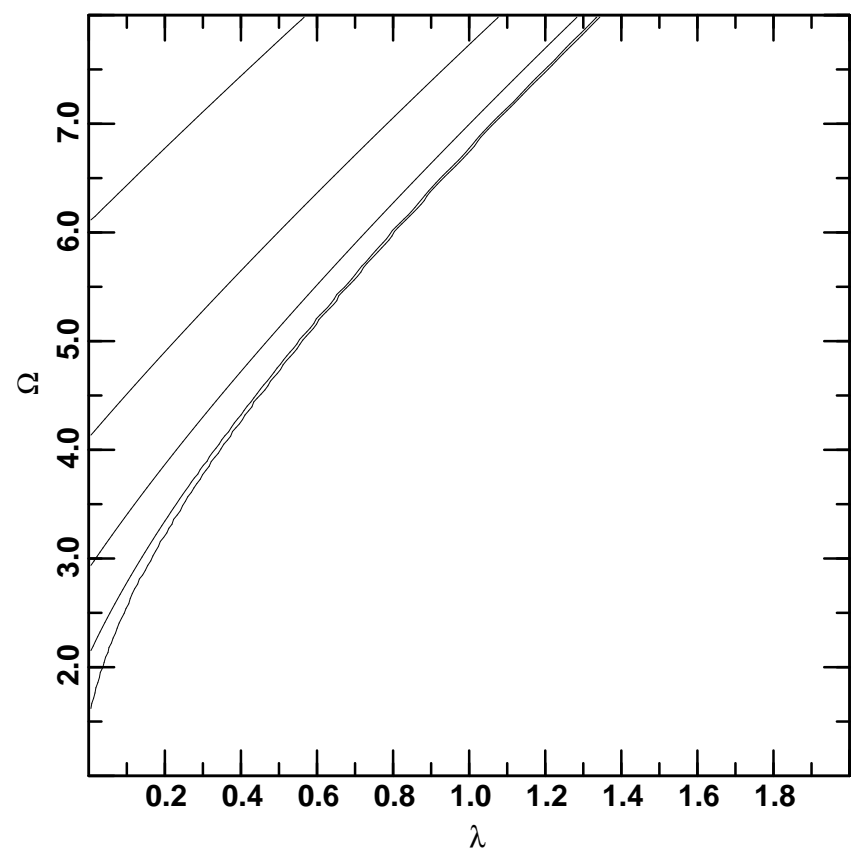

Figure 5. The age of the universe as a fraction of the time between the big bang and maximum expansion. Contours, from the upper left, are at 0.5 , $0.4,0.3,0.2$ and 0.1 .

time of maximum expansion, $\lambda$ and $\Omega$ are infinite since $H=0$ ). Fig. 5 shows the same for $\lambda>0$. While the constraints aren't quite as strong here, in the Section 5 I show that a different argument renders many of the models in this part of parameter space unlikely.

Note that this argument is completely independent of $H_{0}$ : whatever the value of $H_{0}$, i.e. whatever the age of the universe, models which recollapse have large values of $\lambda$ and $\Omega$ for only a relatively short time.

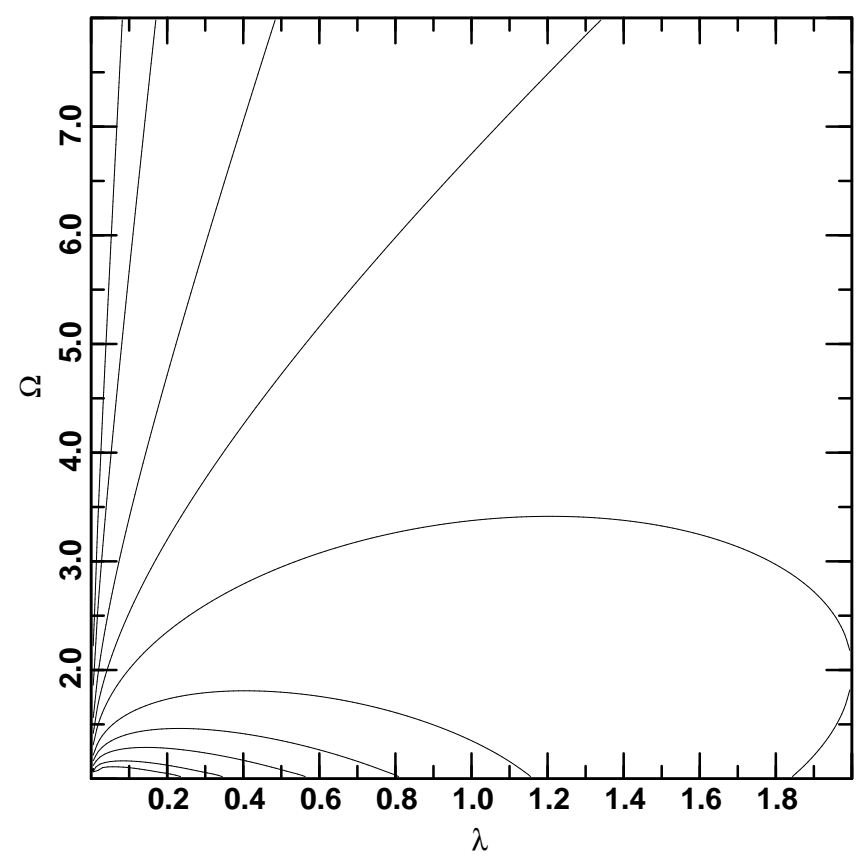

Figure 6. The constant of motion $\alpha$ (see equation [10). From upper left to lower left, contours are at $0.1,0.2,0.5,1,2,5,10,20,50$ and 100.

\section{COSMOLOGICAL MODELS WHICH EXPAND FOREVER: REVERSING THE FINE-TUNING PROBLEM}

An interesting point has been made by Lake (2005), though it is implicit in Stabell \& Refsdal (1966): there are many cosmological models which expand forever in which, although $\lambda$ and $\Omega$ evolve with time, they never stray very far from their initial values. In particular, there are many models for which $K$ is roughly 0 today and which never deviate very far from this value. Looking at $K$ rather than just $\Omega$, these models don't suffer from a flatness problem in the restricted sense, though as mentioned above the same arguments apply to $\Omega$ (and analogously to $\lambda$ ) as apply in the $\lambda=0$ case, i.e. they still suffer from the Einstein-de Sitter problem. Nevertheless, if, as observations suggest, $K \approx 0$ and $0<\lambda_{0}<1$, then large values of $\lambda$ and $\Omega$ never occur during the evolution of the universe. However, this is not really a satisfactory solution, since it assumes the observed values of $\lambda_{0}$ and $\Omega_{0}$ rather than explaining why we observe these or similar values and the Einstein-de Sitter problem still exists (especially in respect to $\Omega$ becoming arbitrarily small in the future).

More interesting is an argument due to Lake (2005) which solves the Einstein-de Sitter problem as well for models with $k=+1$ which will expand forever. (For models which will expand forever, large values of $\lambda$ and $\Omega$ are possible only for $k=+1$.) Trajectories in the $\lambda-\Omega$ plane have a constant of motion given by equation $103^{3}$ It seems natural to distinguish cosmological models on the basis of their value of $\alpha$. Large values of $\lambda$ and $\Omega$ are possible only for $\alpha \lesssim 1$. This is shown in Fig. 6 (Note that, for clarity, only $\Omega>1$ is shown!) It is obvious that $\alpha \leq 1$ is a necessary condition for having infinitely large values of $\lambda$ or $\Omega$. Already

\footnotetext{
3 This makes it much easier to plot trajectories than by calculating $\lambda$ and $\Omega$ as a function of time, though of course the latter is necessary if one is interested in the amount of time spent during a particular section of the trajectory.
} 
for $\alpha=2$ the maximum value of $\lambda$ is just 2 (for $\Omega=2$ ) and the maximum value of $\Omega$ is $\approx 3.5$ (for $\lambda=1.25$ ).

In this case, the fine-tuning argument is reversed; only in the case of fine-tuning do $\lambda$ and $\Omega$ become arbitrarily large. [If $\alpha$ is seen as a free parameter which can take on any value between $-\infty$ and $\infty$, then a 'random' value would probably be arbitrarily large, corresponding to $K \approx 0$ to a very good approximation. Interestingly, this is what observations seem to indicate: $K_{0} \approx 0$ to within (by now quite good) observational accuracy, but with no specially interesting values for $\lambda_{0}$ or $\Omega_{0}$. For $K_{0} \approx 0$ and $\lambda>0, \lambda$ and $\Omega$ evolve from $(0,1)$ (the Einstein-de Sitter model) to $(1,0)$ (the de Sitter model, like the Einstein-de Sitter model a fixed point, though an attractor rather than a repulsor) without any large values of $\lambda$ or $\Omega$ along the way; for $\lambda<0$ (in which case the universe always recollapses), large values do occur but, as shown above, only during a relatively short time during the history of the universe.] This demonstrates quantitatively that there is no quantitative flatness problem regarding arbitrarily large values of $\lambda$ or $\Omega$ for models which expand forever. [Some models which recollapse but which have $\lambda>0$ also have $\alpha \approx 1$; this provides an additional argument against the existence of the flatness problem in these models which complements that made in Section 4 (where, for $\lambda \approx 0$, that argument is somewhat weaker).] This argument is also independent of the value of $H_{0}$. However, all non-empty models which expand forever asymptotically approach the de Sitter model at $(\lambda, \Omega)=(1,0)$. Thus, one final aspect of the quantitative flatness problem remains: $\Omega$ can become arbitrarily small. This is investigated in the next section.

If we want to use the tightrope-walker analogy to examine large values of $\lambda$ and $\Omega$ in models which expand forever, we can think of the ground representing arbitrarily large values of $\lambda$ and $\Omega$. However, in this case the tightrope walker is secured by a safety line of finite length, which prevents him from reaching the ground (and, as he swings back up after his fall, causes the values of $\lambda$ and $\Omega$ to decrease). The only way he can reach the ground is in the finely tuned case that his rope is long enough, i.e. infinitely long (since the ground corresponds to arbitrarily large values of $\lambda$ and $\Omega)$.

Another class of models which expand forever are the bounce models in which the universe contracts from infinity to a finite size before expanding forever. For those near the $\alpha=1$ curve [the A2 curve in Stabell \& Refsdal (1966)], the same arguments apply as for those on the other side of the curve: only for $\alpha \approx 1$ are large values of $\lambda$ and $\Omega$ possible for a significant period of time. These models begin at the de Sitter model with $\lambda$ and $\Omega$ increasing to $\infty$ in a finite time and return along the same trajectory in the $\lambda-\Omega$ parameter space, in this respect similar to the models which collapse in the future, thus the argument against the flatness problem is similar in the two cases. To be sure, these models have an infinite extent in time in both directions, so in this sense there is no flatness problem (or, more precisely, no 'de Sitter problem', analogous to the Einstein-de Sitter problem discussed above) since they are almost always arbitrarily close to the de Sitter model. If we choose starting values for $\lambda$ and $\Omega$ which are not arbitrarily close to the de Sitter model, then the argument is completely analogous to that for collapsing models.

\section{COSMOLOGICAL MODELS WHICH EXPAND FOREVER: THE WEAK ANTHROPIC PRINCIPLE}

I have now covered the entire $\lambda-\Omega$ parameter space except for bigbang models with (a) $q<0$ (which implies $\lambda>0$ ) and (b) $\Omega$ less than $\approx 2$ (all three values of $k$ are possible) and shown that in all cases there is no flatness problem. What about this remaining portion of parameter space? Models here all have $K \approx 0$ and approach the de Sitter model asymptotically. This means that there is no flatness problem in the restricted sense, as pointed out by Lake (2005). However, $\Omega$ becomes arbitrarily small (and $\lambda$ arbitrarily close to 1 ). Thus, there is still a problem in that we do not observe such values, even though they exist for almost the entire (infinite) lifetime of the universe.

This is essentially the question 'if the universe lasts forever, then why are we near the beginning?' Note that this question could be asked at any time. One could leave it at that and say that since any finite age is arbitrarily close to the beginning, there is nothing special about our time and thus no flatness problem in the timescale sense (i.e. the quantitative flatness problem, why is $\Omega$ not arbitrarily small today).

One can do better by invoking the weak anthropic principle: cosmological parameters can be observed only to have values which permit the existence of cosmologists. For the first time, the analysis is not independent of $H_{0}$. In the de Sitter model, $H$ is constant in time. Hence, in models which asymptotically approach the de Sitter model, $H$ approaches a constant value (which is not that different from $H_{0}$ ). If $H$ is similar to the observed value of $H_{0}$, then small values of $\Omega$ (and values of $\lambda$ near 1) occur only in the relatively distant future of the universe. It is difficult to estimate the probability that cosmologists exist at such future times, but it is clear that humans in their present form probably won't exist. In this sense, we don't observe an arbitrarily small value of $\Omega$ since we probably wouldn't exist in such a universe. This is due not to the small value of $\Omega$ itself but rather to the fact that in the far future there will be no main-sequence stars etc. While this is only as satisfactory as any use of the weak anthropic principle, (a) this does not mean that the argument is invalid, (b) it is needed only to rule out arbitrarily small values of $\Omega$ (and not arbitrarily large ones, which are ruled out by other arguments above) and, (c) as pointed out by Lake (2005), in the restricted sense there is no flatness problem (i.e. $K \approx 0$ during the entire lifetime of the universe). Fig. 7 illustrates this: only arbitrarily old models are arbitrarily close to the de Sitter model. One can see that arbitrarily small values of $\Omega$ (or values of $\lambda$ arbitrarily close to 1 ) occur only when $H t$ approaches $\infty$.

Since the quantity $H t$ is determined by $\lambda_{0}$ and $\Omega_{0}$, one could have an arbitrarily young universe arbitrarily close to the de Sitter model if $H_{0}$ were arbitrarily large. However, such a universe will spend only a short time near the Einstein-de Sitter model, which probably means that structure, and hence cosmologists, would not form in such a universe.

[Since $H t$ has a finite value (namely 1 ) for $\lambda=0$ and $\Omega=0$ and, for $H$ near the observed value of $H_{0}$, the age of the universe for $\Omega=0$ would be approximately the same as the observed age, one might get the impression that, for $\lambda=0$, observing an arbitrarily small value of $\Omega_{0}$ wouldn't be that unlikely. However, this is the case for a universe which is exactly described by the Milne model, which means that it is empty and hence contains no cosmologists. The situation is somewhat different when considering the Milne model as the asymptotic value of a universe with $\lambda=0$ but $\Omega>0$. For $\lambda=0$ and $0<\Omega<1$, the universe approaches 


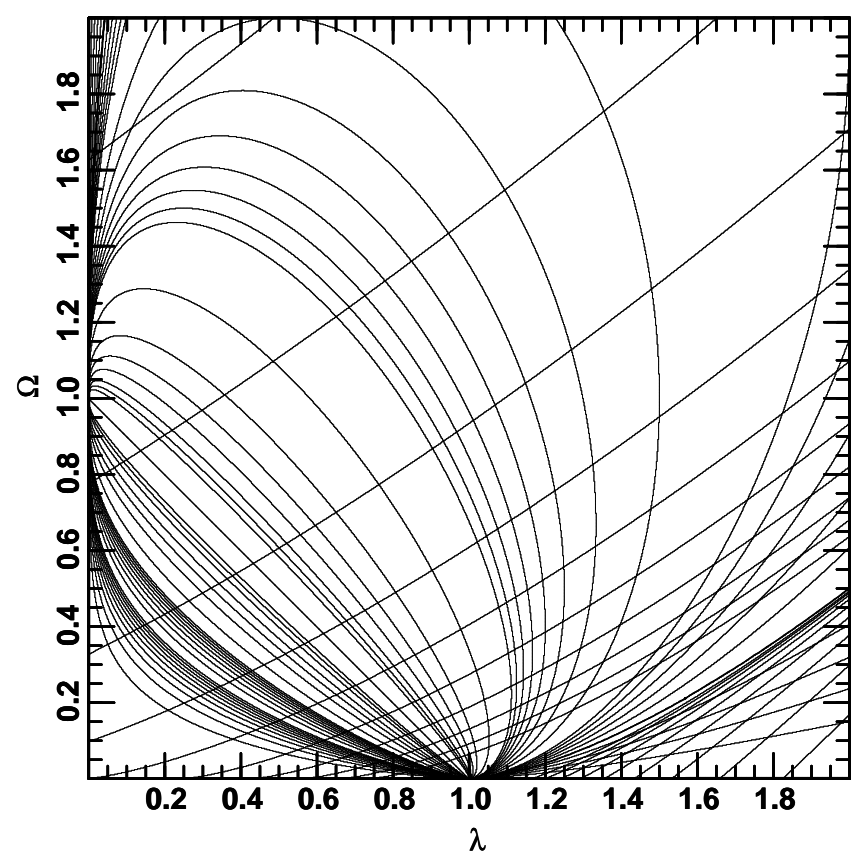

Figure 7. Evolutionary trajectories superposed on contours of constant $\mathrm{Ht}$. Starting at the lower right and moving along a curve perpendicular to the trajectories, contours for $\alpha$ are at $0.2,0.4,0.6,0.8,1.0,1.2,1.4,1.6,1.8,2$, $3,4,5,6,7,8,9,10,20,50,100,200,500,1000$ and 2000. The straight diagonal line for $K=0$ corresponds to $\alpha=\infty$. Continuing below this line, the order is reversed. From upper left to lower right, contours for $\mathrm{Ht}$ are $0.6,0.7,0.8,0.9,1.0,1.1,1.2,1.3,1.4,1.5, \infty$ (which corresponds to $\alpha=1), 1.5,1.4,1.3,1.2,1.1,1.0,0.9,0.8,0.7$. Those to the right of $\infty$ measure the time since the universe was at its minimum size.

the Milne model asymptotically and $H$ approaches 0 . In this case, an arbitrarily small $\Omega$ is reached only after an arbitrarily long time, so a similar argument applies as in the case for $\lambda>0$ : if $H$ now is near the observed value of $H_{0}$, then this happens in the far future when there are no more main-sequence stars etc. On the other hand, if one assumes an age near the current observed age but with an arbitrarily small value of $\Omega$, then this implies that the universe spent only a relatively short time near the Einstein-de Sitter model and hence structure formation would be difficult.]

If we want to use the tightrope-walker analogy to examine the behaviour at arbitrarily large times in models which expand forever, we can think of the ground representing the de Sitter model. After the tightrope walker falls off, he falls towards the ground, but his speed slows with time, so that he never actually reaches the ground.

\section{SUMMARY}

The qualitative flatness problem, i.e. the puzzle why the universe was arbitrarily close to the Einstein-de Sitter mode 4 at early times, does not exist. It is merely a consequence of the way $\lambda$ and $\Omega$ are defined. Neither does the quantitative flatness problem exist: although the cosmological parameters in general evolve with time, it is not puzzling that we don't observe extreme values for them today. In the case of models which will collapse in the future this is because large (absolute) values of $\lambda$ and $\Omega$ occur only during a relatively short time in the lifetime of such a universe, namely

4 Or, for an empty universe, the Milne or de Sitter model. near the time of maximum expansion. $\lambda$ and $\Omega$ can become large only when $H$ becomes small, and this happens only during the time when the universe is at or near its maximum size. [Arbitrarily small (absolute) values, if they occur at all, also occur for only a relatively short time]. For models which will expand forever, large values are possible only for $k=+1$. However, this occurs only for $\alpha \approx 1$. In this case, the fine-tuning argument is reversed; only in the case of fine-tuning do $\lambda$ and $\Omega$ become arbitrarily large. Since all models which will expand forever asymptotically approach $\Omega=0$, arbitrarily small values of $\Omega$ can occur. Those with $\lambda=0$ (and hence $k=-1$ ) approach the Milne model with $\Omega=0$; models with $\lambda>0$, whatever the value of $k$, approach the de Sitter model with $\lambda=1$ (the Milne and de Sitter models themselves are of course stationary points). (If $\lambda=0$ at any time then $\lambda=0$ at all times. Otherwise, arbitrarily small values of $\lambda$, if they occur at all, occur only for a relatively short time.) However, if $H_{0}$ has a value similar to or smaller than the observed value, small values of $\Omega$ will occur only in the far future when anthropic arguments probably make the observation of such a low value of $\Omega$ unlikely. While (for $\lambda>0$ ) a higher value of $H_{0}$ would allow a low value of $\Omega$ even for an age near the observed age, such a universe would have spent only a very short time during which $\Omega$ was not very small, so structure formation would have been strongly suppressed.

It is interesting to note that the three arguments presented here make it unlikely that we would observe extreme values of $\lambda_{0}$ or $\Omega_{0}$. This automatically solves the so-called coincidence problem, which has been called deeply puzzling (e.g. Tegmark, Vilenkin \& Pogosian 2005).

Also interesting is that in the appendix to his seminal paper, Guth (1981) anticipates much of the subsequent discussion. He points out that even though essentially all cosmological models begin arbitrarily close to the Einstein-de Sitter universe (the qualitative flatness problem), this still leaves the question as to why the universe is so close to the Einstein-de Sitter universe today (the quantitative flatness problem). He also rejects the idea that some basic principle must force the universe to conform exactly to the Einstein-de Sitter model on the grounds that this is obviously only an approximation in the case of the real universe (see the discussion of this above). He basically recasts the flatness problem as the longevity problem: fine tuning is required in order that the universe does not recollapse or thin out to extremely low density within a very short time. However, this argument relies on an assumption for the value of $H_{0}$, while our arguments do not need this assumption except in the third category. Also, as I have shown here, even in an extremely short-lived universe (which of course recollapses), extreme values of $\lambda$ or $\Omega$ are observed only during a relatively small fraction of the lifetime of the universe.

If there is no flatness problem, what does this mean for inflation? If there is no flatness problem (or, indeed, even if there were no motivation at all for inflation), this does not mean that inflation could not have occurred. However, it does mean that inflation should not be taken as a given based on the belief that it explains away the flatness problem and thus without it classical cosmology leads to absurd conclusions. Inflation also solves the monopole and isotropy problems. However, the monopole problem seems to be more a problem with theories of particle physics than with cosmology (Narlikar \& Padmanabhan 1991) while Barrow (1995) claims that there is no isotropy problem 5 If all of these claims are true, 5 It is, however, debatable whether Barrow's conclusion is as general as he
claims or depends too strongly on his assumptions. 
then this still does not prove that inflation didn't happen, but the necessity for inflation or something like it is weakened if not destroyed altogether.

\section{Acknowledgments}

I thank Nils Bergvall, Léon Koopmans, Rolf Stabell and Erik Zackrisson for comments on the manuscript. Figures were produced with the GRAL software package written by Rainer Kayser.

\section{REFERENCES}

Barrow J. D., 1995, Phys. Rev. D, 51, 3113

Coles P., 2009, The Cosmic Tightrope, http://telescoper.wordpress.com/2009/05/03/the-cosmic-tightrope/

Coles P., Ellis G. F. R., 1997, Cambridge Lecture Notes in Physics, No. 7, Is the Universe Open or Closed? Cambridge Univ. Press, Cambridge

Dicke R. H., Peebles P. J. E., 1979, in Hawking S. W., Israel W., eds, General Relativity: An Einstein Centenary Survey. Cambridge Univ. Press, London, p. 504

Eddington A. S., 1930, MNRAS, 90, 668

Evrard G., Coles P., 1995, Classical and Quantum Gravity, 12, L93

Guth A. H., 1981, Phys. Rev. D, 23, 347

Helbig P., 1996, ANGSIZ User's Guide, preprint (arXiv:astro$\mathrm{ph} / 9603028 \mathrm{v} 3$ )

Kayser R., Helbig P., Schramm T., 1997, A\&A, 318, 680

Lake K., 2005, Phys. Rev. Lett., 94, 201102

Leahy J. P., 2003, Solutions of the Friedman Equation, http://www.jb.man.ac.uk/ jpl/cosmo/friedman.html

Narlikar J. V., Padmanabhan T., 1991, ARA\&A, 29, 325

Stabell R., Refsdal S., 1966, MNRAS, 132, 379

Tegmark M., Vilenkin A., Pogosian L., 2005, Phys. Rev. D, 71, 103523

This paper has been typeset from a $\mathrm{T}_{\mathrm{E}} \mathrm{X} / \mathrm{LT}_{\mathrm{E}} \mathrm{X}$ file prepared by the author. 